\title{
Treatment strategy for skin and soft tissue infections caused by nontuberculous mycobacteria following various procedures
}

Jae Young Bae, In Sik Yun, Tai Suk Roh, Young Seok Kim

Department of Plastic and Reconstructive Surgery, Institute for Human Tissue Restoration, Gangnam Severance Hospital, Yonsei University College of Medicine, Seoul, Korea
Background The early diagnosis and treatment of skin and soft tissue infections caused by nontuberculous mycobacteria (NTM) are important, especially as infections with rapidly growing mycobacteria (RGM) are rare and difficult to diagnose and treat. Recently, we identified 22 cases of NTM infections; in this study, we suggest treatment strategies by analyzing the demographic characteristics and treatment progress of these patients.

Methods A retrospective study of patients with NTM infections from 2009 to 2019 was conducted. To identify NTM infections, acid-fast bacillus (AFB) staining, Gram staining, polymerase chain reaction (PCR), and cultures of mycobacteria were performed. Empirical treatment with a combination of antibiotics or surgery was performed; species identification and drug susceptibility tests were performed by the Korean National Tuberculosis Association. The final regimen was determined after obtaining the test results.

Results The mean incubation time of NTM was $4.32 \pm 2.88$ weeks. RGM were detected in mycobacterial cultures in 21 of the 22 NTM patients. The results of AFB staining were negative in all patients, although PCR was positive for NTM in one patient. Fourteen patients were hospitalized for treatment with intravenous antibiotics and surgery. Treatment with a combined regimen of oral antibiotics was maintained for a mean of $5.41 \pm 1.85$ months.

Conclusions The unusual clinical manifestations of skin and soft tissue infections caused by NTM make them difficult to diagnose. Suspicion of NTM based on clinical presentation and a detailed examination should be followed by proper treatment involving multiple antibiotics and surgery in these patients.

Keywords Nontuberculous mycobacterium / Skin and soft tissue infection / Treatment strategy
Received: Sep 3, 2020 Revised: Oct 13, 2020 Accepted: Oct 28, 2020 Correspondence: Young Seok Kim Department of Plastic and Reconstructive Surgery, Gangnam Severance Hospital, Yonsei University College of Medicine, 211 Eonju-ro, Gangnam-gu, Seoul 06273, Korea Tel: +82-2-2019-3422, Fax: +82-2-2019-4881, E-mail: psyskim@yuhs.ac

Copyright () 2021 The Korean Society for Aesthetic Plastic Surgery.

This is an Open Access article distributed under the terms of the Creative Commons Attribution Non-Commercial License (https://creativecommons.org/licenses/by-nc/4.0/) which permits unrestricted non-commercial use, distribution, and reproduction in any medium, provided the original work is properly cited. $\quad w w w . e-a a p s . o r g$

\section{INTRODUCTION}

Nontuberculous mycobacteria (NTM) are defined as mycobacteria that do not cause tuberculosis or leprosy. More than 170 species have been classified as NTM based on genetic analyses [1]. They have traditionally been categorized into rapidly growing mycobacteria (RGM) or slowly growing mycobacteria based on their growth rate. Based on the Runyon classification system, RGM include $M y$ cobacterium fortuitum, Mycobacterium chelonae, Mycobacterium abscessus, Mycobacterium peregrinum, Mycobacterium smegmatis, 
and Mycobacterium mucogenicum [2].

RGM species mostly mature within 7 days, compared with 2-4 weeks for most mycobacteria. Skin and soft tissue infections are commonly associated with RGM and they may exhibit localized or regional patterns. Such infections are sometimes encountered in immunosuppressed patients [3] and may develop after accidental inoculation with RGM during trauma, surgery, injection, or cannulation [4]. NTM infections have also been reported after several cosmetic surgical procedures, including rhytidectomy, liposuction, and breast augmentation [5-7].

The cause of NTM infection is unknown in most cases because NTM are not commensals, but instead are bacteria that are commonly present in the environment, including water. NTM grow and are protected within biofilms; this makes NTM resistant to common disinfectants [8]. For this reason, contamination of the tap water system with NTM is possible, and these bacteria can grow inside reusable catheters, needles, or cannulas.

Although early recognition, empirical treatment, and identification are essential to combat NTM infections, a proper diagnosis tends to be delayed because skin and soft tissue infections attributed to NTM are rare in most settings. We identified 22 cases of skin and soft tissue infections caused by NTM after various invasive procedures, including breast augmentation with implants, thread facelift, fat grafting, and intramuscular injections. In this study, we analyzed the following data associated with these patients: demographic characteristics, clinical manifestations, mycobacteriology, and treatment modalities. Based on these analyses, we proposed a protocol for the management of NTM infections in the skin and soft tissue.

\section{METHODS}

\section{Patients}

A retrospective study of patients with skin and soft tissue infections caused by NTM was conducted for the period of 2009 to 2019 at the Gangnam Severance Hospital after obtaining approval from the Institutional Review Board of Gangnam Severance Hospital, Yonsei University Health System (IRB No. \#3-2020-0145). A total of 22 patients with skin and soft tissue infections caused by NTM after various invasive procedures, such as breast augmentation with implants, thread facelifts, fat grafting, and intramuscular injections, were included in this study. The following data of the patients were analyzed: demographic characteristics, clinical manifestations, diagnostic laboratory results, and treatment data. Information included the following: sex, age, location of the infection, procedures performed, symptoms, duration from the point of infection to symptom occurrence, duration from the symptom occurrence to hospital visit, bacterial study findings, histopathological findings, antimicrobial treatment, treatment period, hospital stay, and surgical interventions.

\section{Identification of organisms}

All specimens submitted for identifying NTM were obtained in the form of tissue samples or serous/purulent discharge samples. NTM infection was determined by performing acid-fast bacillus (AFB) staining and Gram staining on the samples; cultures of mycobacteria were also performed. Polymerase chain reaction (PCR) tests were performed in all cases as well. Cultures were observed during a 6-week incubation before being considered negative. In South Korea, when NTM are identified in culture samples from any laboratory at a medical institution, the specimen must be sent to the Korean National Tuberculosis Association (KNTA). Species identification and drug susceptibility tests are conducted by the KNTA and the results are forwarded to the respective institution 2 to 3 weeks later.

\section{Management protocol}

As the identification of NTM species takes over 4 weeks, patient management with empirical antibiotic treatments is mandatory during this period. Empirical treatment was initiated with oral antibiotics, including clarithromycin, moxifloxacin, doxycycline, or sulfamethoxazole/trimethoprim. The use of parenteral antibiotics was determined based on the severity of the initial symptoms or the patient's response to oral antibiotics. The patients were hospitalized with a high dose of cefoxitin and amikacin for 3-4 weeks if treatment with parenteral antibiotics was required. In most cases, incision, drainage, and debridement were performed. As much as possible, the use of extensive debridement was avoided. As per the protocol commonly used, foreign bodies, including implants or thread remnants, were removed as early as possible. If general anesthesia was required for foreign body removal or wide debridement, the patient was hospitalized. The final regimen and duration of the antibiotic therapy varied from patient to patient based on the identified bacterial species and drug susceptibility reports. Anti-tuberculous treatments were maintained for more than 3 months, even if the condition of the wounds showed improvement.

\section{RESULTS}

All patients developed symptoms of infection after undergoing various procedures at local clinics. They were referred to our hospital when there was no amelioration of the symptoms, despite the negative bacterial culture and the standard infection treatment over a period of several weeks. The mean ( \pm standard deviation [SD]) age of the patients was $44.86 \pm 14.11$ years. Infection was identified in the following contexts: one patient (4.5\%) showed signs of infection after a fat graft in the forehead; two patients (9\%) developed symptoms of infection after thread lift on the face; four patients (18.2\%) developed signs of infection after augmentation mammoplasty; one patient (4.5\%) became infected after a fat graft in the breast; one patient (4.5\%) showed signs of infection after excision 
of a mass in the thigh; seven patients (32\%) developed symptoms of infection after intramuscular injection of antibiotics into the buttock; and six patients (27.3\%) developed signs of infection after cannulation in the trunk. Most of the symptoms of infection were discharge, erythema, swelling, and tenderness. It took a mean $( \pm S D)$ of $4.32 \pm 2.88$ weeks from the date of initial procedure or surgery to the date of symptom occurrence and a mean $( \pm \mathrm{SD})$ of $6.82 \pm 7.40$ weeks from the date of symptom occurrence to the date of the hospital visit (Table 1).

In the mycobacterial culture test, NTM were detected in all patients. In contrast, the results of conventional bacterial culture, AFB staining, and Gram staining were negative in all patients. PCR testing detected NTM in only one patient. Species identification tests revealed the presence of $M$. abscessus in 15 patients (68.3\%), $M$. massiliense in three patients (13.6\%), M. fortuitum in three patients (13.6\%), and M. lentiflavum in one patient (4.5\%) (Table 2). All species were RGM except $M$. lentiflavum.

Patients in need of intravenous (IV) antibiotics or surgery under general anesthesia were hospitalized. A total of 14 patients (63.6\%) were hospitalized. Among them, 10 patients (71.4\%) were treated with IV amikacin-cefoxitin combination therapy, three patients (21.4\%) were treated with IV amikacin-imipenem combination therapy, and one patient (7.2\%) was treated with IV amikacin-metronidazole combination therapy. A total of 15 patients underwent surgical treatment, including incision and drainage (four patients, 26.6\%), debridement (five patients, 33.3\%), and foreign body removal (six patients, $40.0 \%$ ). In one patient, breast implants were removed at a private clinic before visiting our hospital.

All patients were treated with oral antibiotics for at least 3 months and the mean $( \pm \mathrm{SD})$ duration of treatment was $5.41 \pm 1.85$ months. In 16 patients (72.9\%) a combination of moxifloxacin and clarithromycin was used as the oral antibiotic regimen. Other antibiotic combinations, including moxifloxacin-doxycycline-azithromycin, clarithromycin-doxycycline-trimethoprim/sulfamethoxazole, azithromycin-amoxicillin/clavulanate, and clarithromycin-levofloxacin were also used based on the patients' conditions (Table 3). Only one patient $(4.5 \%)$ was treated with clarithromycin monotherapy. The oral antibiotic regimen was selected in accordance with the advice of physicians specializing in infectious diseases who made the decision based on the reports of antibiotic susceptibility tests.

Table 1. Patients' demographic characteristics

\begin{tabular}{|c|c|c|c|c|c|c|}
\hline Patient No. & $\begin{array}{c}\text { Sex/age } \\
\text { (yr) }\end{array}$ & Location & Cause & Symptoms & $\begin{array}{c}\text { Duration from the point } \\
\text { of infection to symptom } \\
\text { occurrence (wk) }\end{array}$ & $\begin{array}{l}\text { Duration from } \\
\text { symptom occurrence } \\
\text { to hospital visit (wk) }\end{array}$ \\
\hline 1 & $F / 56$ & Face & Thread lift & Erythema, swelling & 4 & 24 \\
\hline 2 & $F / 42$ & Face & Thread lift & Erythema, swelling & 4 & 24 \\
\hline 3 & $\mathrm{~F} / 39$ & Forehead & Fat graft & Tenderness, swelling & 1 & 4 \\
\hline 4 & $\mathrm{~F} / 31$ & Breast & Fat graft & Seroma, tenderness, heating sense & 6 & 20 \\
\hline 5 & $F / 28$ & Breast & Augmentation mammoplasty & Seroma & 4 & 12 \\
\hline 6 & $F / 34$ & Breast & Augmentation mammoplasty & Discharge, tenderness & 5 & 12 \\
\hline 7 & $F / 39$ & Breast & Augmentation mammoplasty & Discharge, erythema, swelling & 12 & 4 \\
\hline 8 & $\mathrm{~F} / 38$ & Breast & Augmentation mammoplasty & Discharge, erythema, swelling & 4 & 6 \\
\hline 9 & $\mathrm{~F} / 53$ & Thigh & Excision & Discharge, erythema, swelling & 3 & 1 \\
\hline 10 & $\mathrm{~F} / 76$ & Trunk & Cannulation & Discharge, erythema, swelling & 1 & 5 \\
\hline 11 & $\mathrm{~F} / 20$ & Trunk & Cannulation & Discharge, erythema, swelling & 10 & 4 \\
\hline 12 & $F / 65$ & Trunk & Cannulation & Discharge, erythema, swelling & 1 & 3 \\
\hline 13 & $F / 49$ & Trunk & Cannulation & Discharge, erythema, swelling & 4 & 5 \\
\hline 14 & $F / 52$ & Trunk & Cannulation & Discharge, erythema, swelling & 8 & 1 \\
\hline 15 & $F / 62$ & Trunk & Cannulation & Discharge, erythema, swelling & 1 & 3 \\
\hline 16 & $M / 36$ & Buttock & IM injection & Discharge, erythema, swelling & 8 & 1 \\
\hline 17 & $\mathrm{~F} / 27$ & Buttock & IM injection & Discharge, erythema, swelling & 5 & 1 \\
\hline 18 & $\mathrm{~F} / 40$ & Buttock & IM injection & Discharge, erythema, swelling & 2 & 3 \\
\hline 19 & $F / 64$ & Buttock & IM injection & Discharge, erythema, swelling & 2 & 3 \\
\hline 20 & $\mathrm{~F} / 40$ & Buttock & IM injection & Discharge, erythema, swelling & 3 & 4 \\
\hline 21 & $\mathrm{~F} / 60$ & Buttock & IM injection & Discharge, erythema, swelling & 3 & 2 \\
\hline 22 & $M / 36$ & Buttock & IM injection & Discharge, erythema, swelling & 4 & 4 \\
\hline
\end{tabular}

$F$, female; $M$, male; IM, intramuscular. 
Table 2. Laboratory examination results

\begin{tabular}{|c|c|c|c|c|c|c|}
\hline Patient No. & $\begin{array}{l}\text { Bacterial } \\
\text { culture }\end{array}$ & Mycobacterial culture & $\begin{array}{c}\text { Acid-fast bacillus } \\
\text { stain }\end{array}$ & Gram stain & PCR & Species identification \\
\hline 1 & - & Nontuberculous mycobacterium & - & - & - & Mycobacterium abscessus \\
\hline 2 & - & Nontuberculous mycobacterium & - & - & - & Mycobacterium abscessus \\
\hline 3 & - & Nontuberculous mycobacterium & - & - & - & Mycobacterium massiliense \\
\hline 4 & - & Nontuberculous mycobacterium & - & - & - & Mycobacterium fortuitum \\
\hline 5 & - & Nontuberculous mycobacterium & - & - & - & Mycobacterium fortuitum \\
\hline 6 & - & Nontuberculous mycobacterium & - & - & - & Mycobacterium fortuitum \\
\hline 7 & - & Nontuberculous mycobacterium & - & - & - & Mycobacterium massiliense \\
\hline 8 & - & Nontuberculous mycobacterium & - & - & - & Mycobacterium lentiflavum \\
\hline 9 & - & Nontuberculous mycobacterium & - & - & - & Mycobacterium massiliense \\
\hline 10 & - & Nontuberculous mycobacterium & - & - & - & Mycobacterium abscessus \\
\hline 11 & - & Nontuberculous mycobacterium & - & - & - & Mycobacterium abscessus \\
\hline 12 & - & Nontuberculous mycobacterium & - & - & - & Mycobacterium abscessus \\
\hline 13 & - & Nontuberculous mycobacterium & - & - & - & Mycobacterium abscessus \\
\hline 14 & - & Nontuberculous mycobacterium & - & - & - & Mycobacterium abscessus \\
\hline 15 & - & Nontuberculous mycobacterium & - & - & - & Mycobacterium abscessus \\
\hline 16 & - & Nontuberculous mycobacterium & - & - & - & Mycobacterium abscessus \\
\hline 17 & - & Nontuberculous mycobacterium & - & - & - & Mycobacterium abscessus \\
\hline 18 & - & Nontuberculous mycobacterium & - & - & - & Mycobacterium abscessus \\
\hline 19 & - & Nontuberculous mycobacterium & - & - & - & Mycobacterium abscessus \\
\hline 20 & - & Nontuberculous mycobacterium & - & - & - & Mycobacterium abscessus \\
\hline 21 & - & Nontuberculous mycobacterium & - & - & - & Mycobacterium abscessus \\
\hline 22 & - & Nontuberculous mycobacterium & - & - & $\begin{array}{c}\text { Nontuberculous } \\
\text { mycobacteria }\end{array}$ & Mycobacterium abscessus \\
\hline
\end{tabular}

PCR, polymerase chain reaction; -, negative result.

Patients' characteristics are summarized in Table 4.

\section{Cases}

Case 1

The patient was a 56-year-old woman with no medical history who underwent bilateral thread lift at a local clinic. One month postoperatively, she developed tenderness and pus-like discharge on both cheeks. Despite multiple incisions and drainage and treatment with conventional antibiotics, the wound did not show improvement. The results of bacterial culture were negative several times. Six months after the manifestation of the initial symptoms, the patient was transferred to our outpatient clinic for further evaluation, diagnosis, and proper treatment. Foreign body (thread remnants) removal and minimal debridement of the granulation tissue were performed under general anesthesia. The obtained tissue specimens were subjected to histopathological examination, Gram staining, bacterial culture, AFB staining, and mycobacterial culture. Empirical antibiotic therapy, comprising intravenous amikacin $(750 \mathrm{mg}$, once daily) and cefoxitin ( $3 \mathrm{~g}$, thrice daily) was initiated immediately; oral clarithromycin (500 mg, twice daily) was also used. Treatment with IV antibiotics was maintained for 4 weeks. Among the various tests, only the culture for mycobacteria showed positive results in 1 week. The specimen was sent to the KNTA, which performed species identification and drug susceptibility tests. Species identification test revealed the pathogen to be $M$. abscessus. Based on the results of the drug susceptibility tests, the oral antibiotic regimen was changed to a combination of moxifloxacin ( $400 \mathrm{mg}$, once daily), doxycycline (100 mg, once daily), and azithromycin (500 mg, once daily). Five months after surgical debridement and multi-antibiotic combination therapy, the wound had improved completely, but the oral antibiotic regimen was maintained for another 3 months. Antibiotic treatment was administered for a period of 8 months to treat the infection in this patient. The patient showed no sign of infection recurrence during the follow-up period, but multiple scars with contracture remained (Fig. 1).

\section{Case 2}

The patient was a 39-year-old woman with no medical history who underwent augmentation mammoplasty with a textured cohesive gel breast implant at a local clinic. Four months postoperatively, she developed erythematous swelling and pus-like discharge on the right breast. The wound became aggravated despite 3 weeks of 
Table 3. Antibiotics and surgical treatment of patients

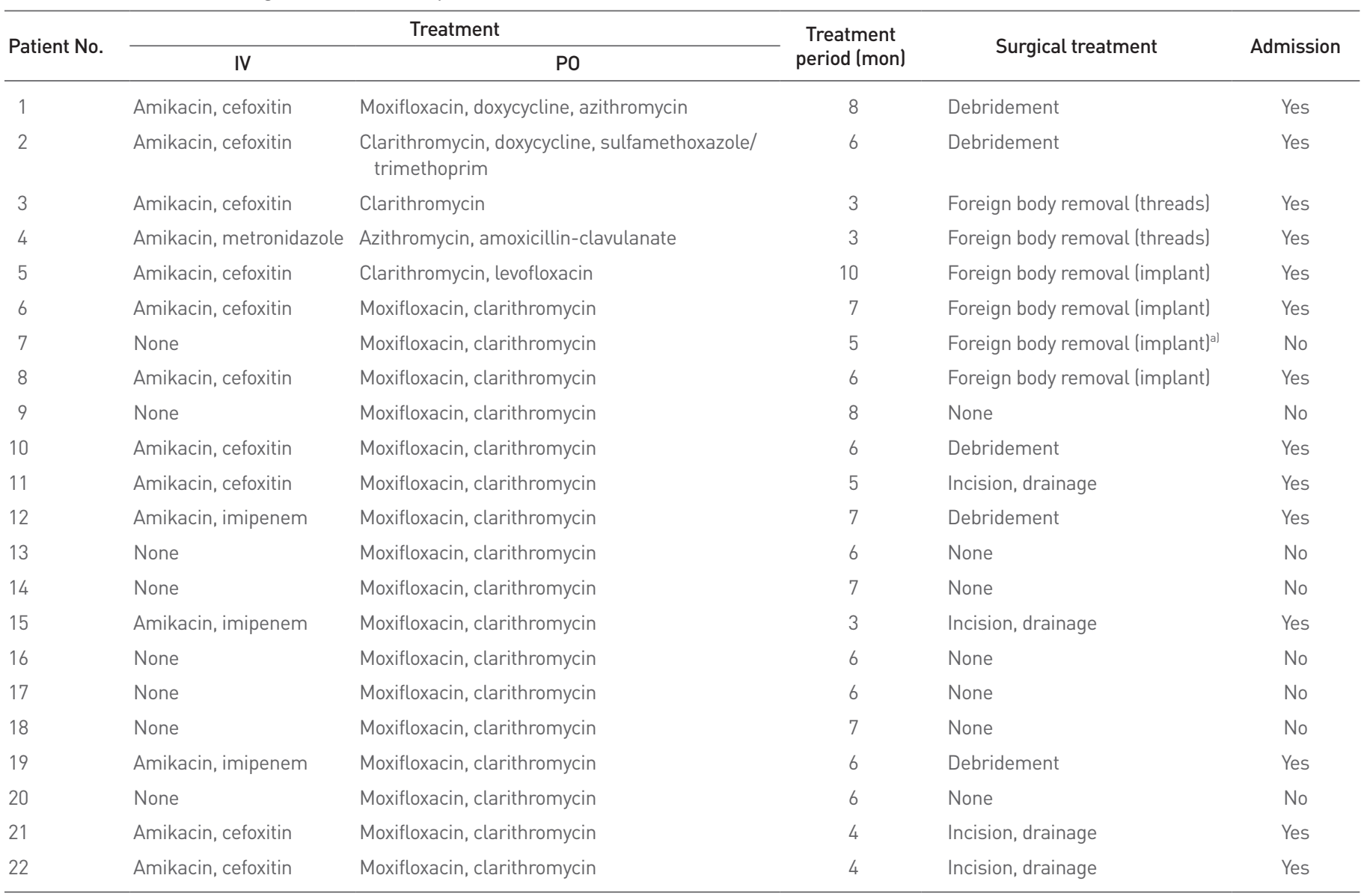

Dosage of antibiotics: amikacin, $750 \mathrm{mg}$ once daily; imipenem, $500 \mathrm{mg}$ four times daily; cefoxitin, $3 \mathrm{~g}$ three times daily; moxifloxacin, $400 \mathrm{mg}$ once daily; clarithromycin, 500 mg twice daily; doxycycline, 100 mg once daily; azithromycin, 500 mg once daily; sulfamethoxazole/trimethoprim, 400 mg/80 mg once daily; amoxicillin-clavulanate, $625 \mathrm{mg}$ thrice daily; levofloxacin, $500 \mathrm{mg}$ once daily.

IV, intravenous; PO, oral.

${ }^{a}$ This patient underwent breast implant removal at a private clinic before visiting our hospital.
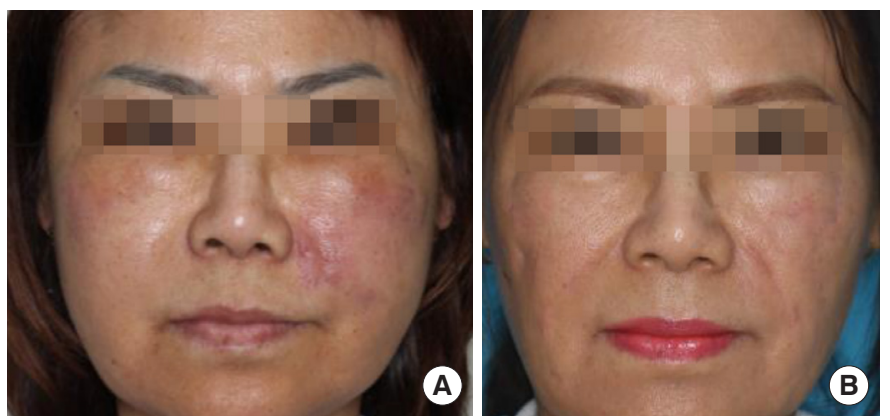

Fig. 1. Case 1. A 56-year-old woman who underwent bilateral thread lift developed a Mycobacterium abscessus infection. The infection healed successfully after surgical debridement, intravenous amikacin and cefoxitin, and oral clarithromycin and moxifloxacin for 6 months. (A) Before treatment. (B) Six months after treatment.

treatment with conventional antibiotics. The results of bacterial culture were negative several times. The patient was transferred to our outpatient clinic for further evaluation, diagnosis, and proper treatment. Intracapsular fluid collection with a sinus tract toward the skin was identified by magnetic resonance imaging. Implant removal and debridement of the granulation tissue were performed under general anesthesia. The obtained tissue specimens were subjected to a histopathological examination, Gram staining, bacterial culture, AFB staining, and mycobacterial culture. Empirical antibiotic therapy, comprising intravenous amikacin $(750 \mathrm{mg}$, once daily) and cefoxitin ( $3 \mathrm{~g}$, thrice daily) was initiated immediately; oral clarithromycin (500 mg, twice daily) was also used. Treatment with IV antibiotics was maintained for 3 weeks. Among the various tests, only the culture for mycobacteria showed positive results in 1 week. The specimen was sent to the KNTA, which performed species identification and drug susceptibility tests. The species identification test revealed the pathogen to be $M$. massiliense. Based on the results of the drug susceptibility tests, the oral antibiotic regimen was changed to a combination of clarithromycin (500 mg, twice daily) and moxifloxacin (400 mg, once daily). One month af- 
Table 4. Patients' characteristics

\begin{tabular}{|c|c|}
\hline Variable & Value $(n=22)$ \\
\hline Age (yr) & $44.86 \pm 14.11(27-76)$ \\
\hline Female sex & 20 (90.9) \\
\hline \multicolumn{2}{|l|}{ Location of lesion } \\
\hline Face & $5(22.5)$ \\
\hline Breast & $3(13.5)$ \\
\hline Trunk & $6(27.0)$ \\
\hline Buttock & 7 (32.5) \\
\hline Thigh & $1(4.5)$ \\
\hline \multicolumn{2}{|l|}{ Cause of infection } \\
\hline Thread lift & $2(9.1)$ \\
\hline Fat graft & $2(9.1)$ \\
\hline Augmentation mammoplasty & $4(18.0)$ \\
\hline Mass excision & $1(4.5)$ \\
\hline Cannulation & $6(27.0)$ \\
\hline Intramuscular injection & $7(32.5)$ \\
\hline $\begin{array}{l}\text { Duration from the point of infection to symptom } \\
\text { occurrence (wk) }\end{array}$ & $4.32 \pm 2.88(1-12)$ \\
\hline $\begin{array}{l}\text { Duration from the symptom occurrence to } \\
\text { hospital visit (wk) }\end{array}$ & $6.82 \pm 7.40(1-24)$ \\
\hline \multicolumn{2}{|l|}{ Species identification } \\
\hline Mycobacterium abscessus & $15(68.5)$ \\
\hline Mycobacterium fortuitum & $3(13.5)$ \\
\hline Mycobacterium massiliense & $3(13.5)$ \\
\hline Mycobacterium lentiflavum & $1(4.5)$ \\
\hline Treatment period (mon) & $5.41 \pm 1.85(3-10)$ \\
\hline
\end{tabular}

Values are presented as mean \pm SD (range) or number (\%).

ter surgical debridement and multi-antibiotic combination therapy, the wound had improved completely, but the oral antibiotic regimen was maintained for another 3 months. The antibiotic treatment was employed for a period of 4 months to treat the infection in this patient. The patient showed no sign of infection recurrence during the follow-up period (Fig. 2).

\section{DISCUSSION}

Outbreaks of NTM infections after plastic surgery have been documented globally in the past. Nine cases of surgical-site RGM infections were reported in Caracas, Venezuela after liposuction and liposculpture. All episodes of RGM infections occurred within 2 months. The median time from the surgical procedure to the onset of symptoms was 15 days. The surgical procedures associated with the development of RGM infections include abdominal liposuction, anterior and posterior thigh liposuction, and bilateral nasolabial fold liposculpture [9]. In seven patients, RGM were confirmed by microbial culture; the species identified were M. chelonae, $M$. fortuitum, and M. abscessus [9]. The Centers for Disease Control
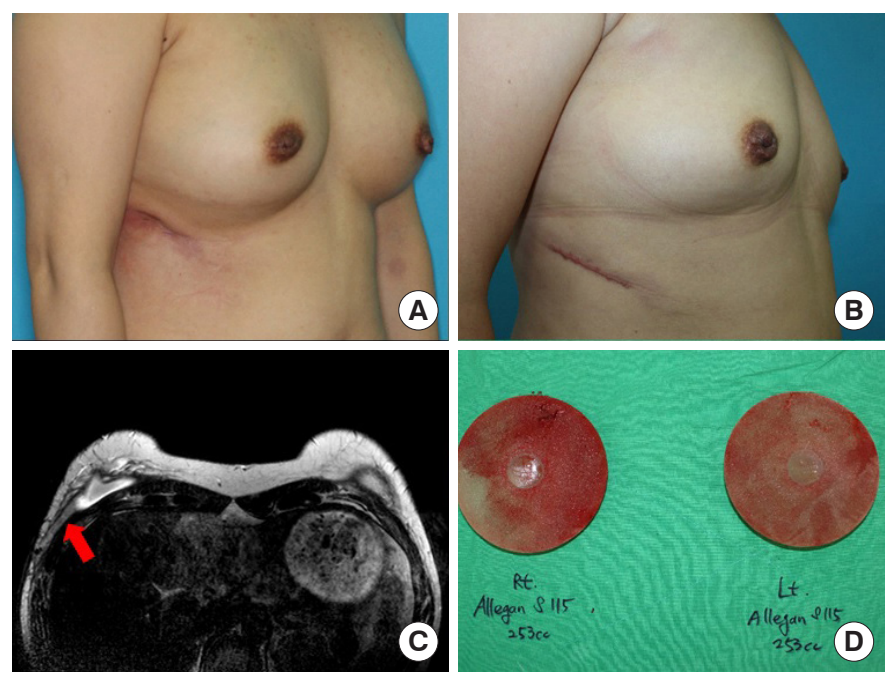

Fig. 2. Case 2. A 39-year-old woman who underwent augmentation mammoplasty developed a Mycobacterium massiliense infection. The infection healed successfully after implant removal, debridement, intravenous amikacin and cefoxitin, and oral clarithromycin for 4 months. (A) Before treatment. (B) Five months after treatment. (C) Magnetic resonance imaging shows intracapsular fluid collection with a sinus tract toward the skin (red arrow). (D) Removed breast implant.

also reported an outbreak of 20 cases of NTM infections among patients who had undergone abdominoplasty in the Dominican Republic and Santo Domingo; all cultured microorganisms were M. abscessus. The symptoms developed 2-18 weeks after the procedures (median interval, 7 weeks), and 19 of the 20 patients were cured after a median of 9 months of combination therapy with antibiotics (range, 2-12 months) [10].

Postsurgical wound infections due to NTM are not limited to plastic surgery. Eyebrow tattooing, acupuncture, laser resurfacing, and many other procedures-including intramuscular injections in our study—can cause NTM infections [11]. In South Korea, 40 patients were infected by NTM after acupuncture at oriental medicine clinic [12].

As NTM infections are challenging to diagnose, early suspicion based on the patient's medical history, surgical history, and unusual clinical features plays as crucial role in diagnosing the condition. Typically, if bacterial infections are transmitted during surgery or procedures, the symptoms develop after approximately $24-72$ hours of bacterial incubation [13]. However, infections caused by NTM generally appear a few weeks to few months after the procedure [12]. Kannaiyan et al. [14] also reported that the incubation period ranged from 20 to 66 days, with a median incubation period of 6 weeks. In this study, it took an average of 4.32 weeks from the time of the procedure for the symptoms to occur. If the wounds do not show improvement despite antibiotic treatment, even after the appearance of late symptoms of infection, one should suspect an un- 
usual infection. Otherwise, the treatment can be delayed until the time of the initial diagnosis. In this study, an average of 6.82 weeks elapsed between the initial appearance of symptoms and patients' presentation to our hospital, and it took 9-10 weeks to make the final diagnosis based on the results of 2 to 3 weeks of mycobacterial culture. NTM infections tend not to cause symptoms associated with systemic inflammation, such as fever, leukocytosis, or lymphadenopathy. Like other clinically common pathogens, such as Staphylococcus aureus or Streptococcus pyogenes, NTM can also cause abscesses, serous fluid collections, cutaneous fistulae, openings, and cellulitis [15]. However, it is noteworthy that the results of conventional bacterial cultures are negative in most cases of NTM infections. Therefore, an NTM infection should be suspected if there is no improvement in the wound following antibiotic treatment for 1 to 2 weeks, even if the results of the bacterial culture are negative.

Once an NTM infection is suspected, it is necessary to initiate empirical antibiotic therapy and proper wound management immediately. The first step is to collect an appropriate specimen, which can be obtained as a tissue sample by debridement or as a fluid sample by aspiration or drainage. For mycobacteria-related tests, the obtained tissue samples should not be placed in transport media with a swab, but instead should be placed in an aseptic test tube. In this study, the results of bacterial culture, Gram staining, and AFB staining were all negative in all patients. Although PCR tests can produce results quickly, they often yield false-negative results. Only one patient was positive for NTM in the PCR test in this study. Thus, mycobacterial culture is the gold standard for confirming the presence of NTM. Usually, the results are obtained within 2 to 3 weeks if the mycobacterial culture is positive. However, slow-growing NTM require at least 6 weeks of culture [16].

As for surgical intervention, which involves debridement and drainage, minimal debridement is recommended. Of course, debridement can reduce the microbial burden. However, in most cases, if the growth of mycobacteria is not controlled by antibiotics, granulation and serous/purulent discharge occur again, and the condition of the wound does not improve. Therefore, we do not recommend extensive or repetitive debridement because doing so only increases the wound size. In cases where a foreign body is embedded-including implants or prostheses-immediate removal is mandatory.

Empirical antibiotic treatment is essential because it takes more than 4 weeks to obtain the results of the species identification and drug susceptibility tests. Our first-line empirical antibiotic regimen included IV amikacin and cefoxitin, as well as oral clarithromycinmoxifloxacin combination therapy based on the susceptibility results of RGM reported by El Helou et al. [17]. El Helou et al. recommended that an initial antimycobacterial combination should ideally include IV amikacin, a quinolone, and a macrolide. Generally, almost all mycobacterial species are susceptible to amikacin. Moreover, M. abscessus is usually susceptible to macrolides, but re- sistant to quinolone. By contrast, M. fortuitum and M. neoaurum, which are usually susceptible to quinolone, are resistant to macrolides, whereas M. mucogenicum is often susceptible to quinolones and macrolides [17].

The antibiotic regimen could be altered based on the result of the drug susceptibility tests. The ideal duration of treatment of skin and soft tissue NTM infections is controversial. For lower respiratory tract infections, antibiotic treatment of up to 12 months might be recommended [17]. We recommend that antibiotic therapy should be maintained for at least 3 months or at least an additional 2 to 3 months after the recovery of the skin and soft tissue wounds. We also recommend consulting with an infectious disease specialist to set a precise endpoint of treatment. In our experience, $M$. $a b$ scessus infections tend to take longer to treat owing to their severe symptoms and poor antibiotic response than infections associated with other NTM species.

Based on previous recommendations and studies, we suggest a treatment strategy at our institution for skin and soft tissue infections caused by NTM, especially RGM, in light of our experiences (Fig. 3). Kim et al. [18] also reported a treatment strategy of strongly suspected NTM infections following aesthetic procedures. However, our institution deployed improved strategies of diagnosis and treatment. First, we suggest mycobacterial culture as the gold standard for confirming NTM, instead of PCR, AFB staining, or histopathology results, which are often ambiguous and inaccurate. Second, when using antibiotics in patients with severe symptoms, we chose IV amikacin, cefoxitin, or imipenem, rather than clarithromycin and moxifloxacin. We also changed the oral antibiotic regimen from clarithromycin and moxifloxacin to the antibiotics to which the pathogen showed the greatest susceptibility, rather than continuing to use the same antibiotics. Third, we kept using antibiotics for at least 2 months, even after symptoms improved and wounds healed.

Some NTM-suspected patients who were referred to our hospital had negative results on NTM culture or PCR after starting empirical antibiotics. In some of these cases, repeated bacterial culture revealed nosocomial pathogens such as methicillin-resistant $S$. aureus, Staphylococcus epidermidis, or diphtheroids, and these patients then received antibiotic treatment according to the susceptibility of the pathogen. In other cases, the wound improved after using empirical antibiotics and persistent wound management without pathogen identification. Therefore, even if the NTM culture is negative, if there is no improvement in the wound, we suggest repeated bacterial or NTM culture while continuing empirical antibiotics and regular wound management.

Our examinations and treatment guidelines are also based on the reports published by the American Thoracic Society and the Infectious Disease Society of America. Their guidelines recommended a combination macrolide-based antibiotic regimen for preventing drug resistance and recurrence. This suggests that drug suscep- 
Previous history of operation with or without instruments/prosthesis

- Breast implant, liposuction, filler injection, facelift, catheter insertion site, etc.

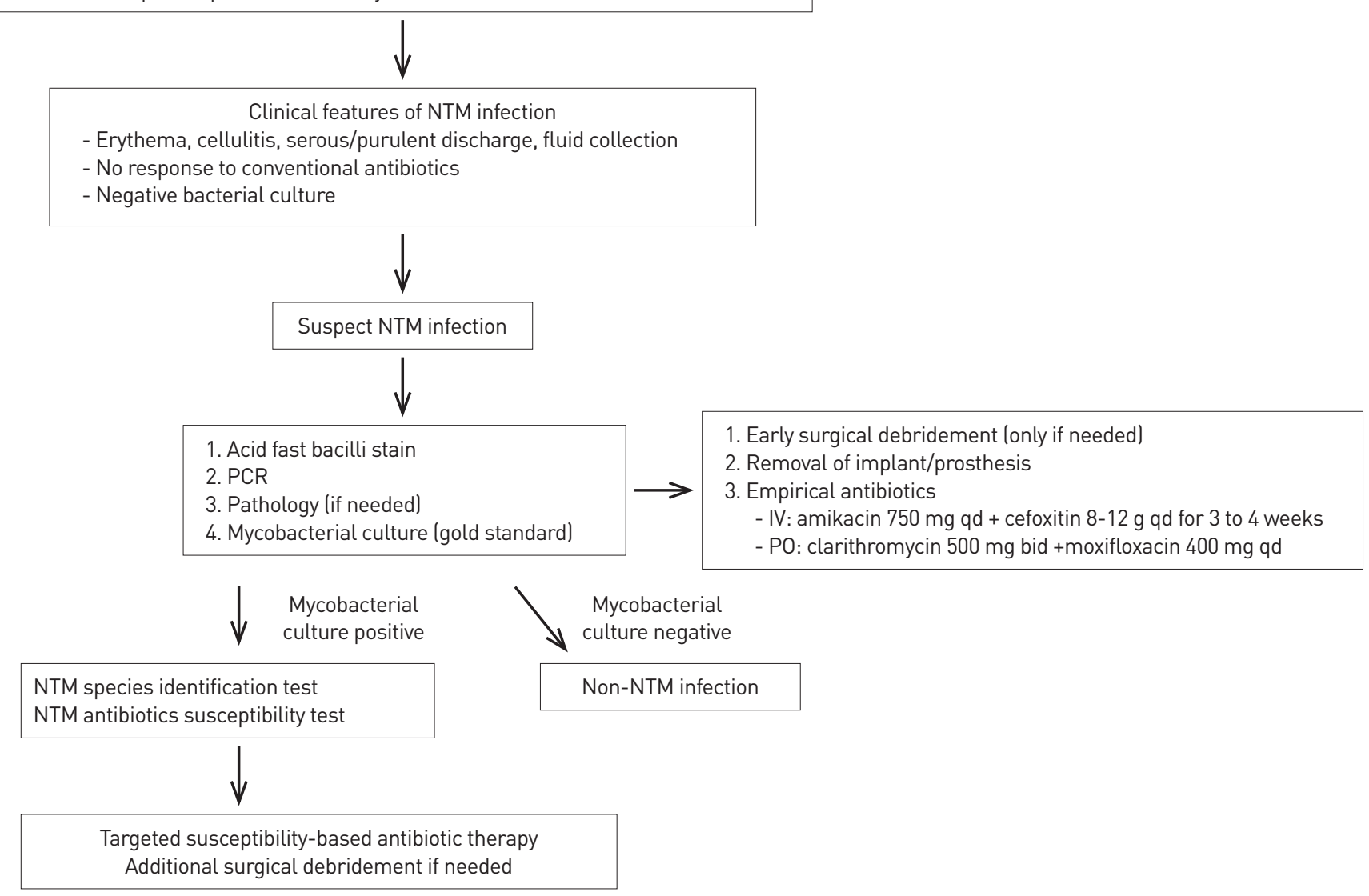

Fig. 3. Diagnosis and treatment strategy used. NTM, nontuberculous mycobacteria; PCR, polymerase chain reaction; IV, intravenous; PO, oral; qd, once daily; bid, twice daily.

tibility testing should be performed for all species of NTM. Antibiotics effective against microbial infections-as part of multidrug regimens-should be administered for longer periods. Surgical intervention including debridement, drainage, and massive irrigation can also be effective against such infections [18,19].

One of the limitations of our study is the lack of diverse cases in the field of plastic surgery; instead, our study included NTM infections caused by intramuscular injection and cannulation. However, procedures in the field of plastic surgery, including filler injection, fat injection, liposuction, tumescent injection, and local anesthetic injections, can cause NTM infections through contaminated water or using an infected cannula. For example, Rodriguez et al. [20] reported NTM infection in subcutaneous tissue of the face after dermal filler injection using a cannula. Macadam et al. [7] also reported cases of breast implant infection that were thought to be caused by contaminated water, skin infections, or instrument infections (including cannulas) in hospitals. In this regard, our experiences of NTM infection caused by intramuscular injection and cannulation provide meaningful evidence for diagnosing and treating NTM in- fections in the field of plastic surgery. The following can serve as sources of NTM infections: contaminated water, aqueous solutions, injectable medications, gentian violet, antiseptic solutions, and inappropriately sterilized surgical devices [21,22]. To identify the cause of infection, a mycobacterial examination should be conducted thoroughly all around the operating environment and epidemiological investigations should be conducted. Water is usually a natural habitat for NTM, and the formation of biofilms plays a key role in their transmission [23].

Because of their unusual clinical manifestations, a high degree of suspicion is necessary to diagnose skin and soft tissue infections caused by NTM, especially in patients with implants or cannulas who do not respond to typical antibiotic treatments. For the management of these patients, it is critical to pay attention to the clinical presentation and the initial integrated and detailed examination, followed by proper treatment with surgical interventions and suitable empirical antibiotics. 


\section{NOTES}

\section{Conflict of interest}

Tai Suk Roh is an editorial board member of the journal but did not involve in the peer reviewer selection, evaluation, or decision process of this article. No other potential conflicts of interest relevant to this article were reported.

\section{Ethical approval}

The study was approved by the Institutional Review Board of Gangnam Severance Hospital (IRB No. \#3-2020-0145) and performed in accordance with the principles of the Declaration of Helsinki.

\section{Patient consent}

The patients provided written informed consent for the publication and the use of their images.

\section{ORCID}

Jae Young Bae

In Sik Yun

Tai Suk Roh

Young Seok Kim https://orcid.org/0000-0002-0258-0606

https://orcid.org/0000-0003-1103-7047

https://orcid.org/0000-0001-8681-159X

https://orcid.org/0000-0002-0981-2107

\section{REFRENCES}

1. Esteban J, Garcia-Pedrazuela M, Munoz-Egea MC, et al. Current treatment of nontuberculous mycobacteriosis: an update. Expert Opin Pharmacother 2012;13:967-86.

2. Yoo SJ, Lee KH, Jung SN, et al. Facial skin and soft tissue infection caused by Mycobacterium wolinskyi associated with cosmetic procedures. BMC Infect Dis 2013;13:479.

3. Horsburgh CR Jr, Selik RM. The epidemiology of disseminated nontuberculous mycobacterial infection in the acquired immunodeficiency syndrome (AIDS). Am Rev Respir Dis 1989;139:4-7.

4. Zhang X, Liu W, Liu W, et al. Cutaneous infections caused by rapidly growing mycobacteria: case reports and review of clinical and laboratory aspects. Acta Derm Venereol 2015;95:985-9.

5. Safranek TJ, Jarvis WR, Carson LA, et al. Mycobacterium chelonae wound infections after plastic surgery employing contaminated gentian violet skin-marking solution. N Engl J Med 1987;317:197-201.

6. Murillo J, Torres J, Bofill L, et al. Skin and wound infection by rapidly growing mycobacteria: an unexpected complication of liposuction and liposculpture. The Venezuelan Collaborative Infectious and Tropical Diseases Study Group. Arch Dermatol 2000;136:1347-52.

7. Macadam SA, Mehling BM, Fanning A, et al. Nontuberculous mycobacterial breast implant infections. Plast Reconstr Surg 2007;119:33744.

8. Gonzalez-Santiago TM, Drage LA. Nontuberculous mycobacteria: skin and soft tissue infections. Dermatol Clin 2015;33:563-77.

9. Centers for Disease Control and Prevention (CDC). Rapidly growing mycobacterial infection following liposuction and liposculpture: Caracas, Venezuela, 1996-1998. MMWR Morb Mortal Wkly Rep 1998; 47:1065-7.

10. Furuya EY, Paez A, Srinivasan A, et al. Outbreak of Mycobacterium abscessus wound infections among "lipotourists" from the United States who underwent abdominoplasty in the Dominican Republic. Clin Infect Dis 2008;46:1181-8.

11. Culton DA, Lachiewicz AM, Miller BA, et al. Nontuberculous mycobacterial infection after fractionated $\mathrm{CO}(2)$ laser resurfacing. Emerg Infect Dis 2013;19:365-70.

12. Song JY, Sohn JW, Jeong HW, et al. An outbreak of post-acupuncture cutaneous infection due to Mycobacterium abscessus. BMC Infect Dis 2006;6:6.

13. Fairbrother RW. A text-book of bacteriology. 4th ed. London: Elsevier Butterworth Heinemann; 1945.

14. Kannaiyan K, Ragunathan L, Sakthivel S, et al. Surgical site infections due to rapidly growing mycobacteria in Puducherry, India. J Clin Diagn Res 2015;9:DC05-8.

15. Green DA, Whittier S, Greendyke W, et al. Outbreak of rapidly growing nontuberculous mycobacteria among patients undergoing cosmetic surgery in the Dominican Republic. Ann Plast Surg 2017;78:1721.

16. Pfyffer GE, Wittwer F. Incubation time of mycobacterial cultures: how long is long enough to issue a final negative report to the clinician? J Clin Microbiol 2012;50:4188-9.

17. El Helou G, Viola GM, Hachem R, et al. Rapidly growing mycobacterial bloodstream infections. Lancet Infect Dis 2013;13:166-74.

18. Kim HR, Yoon ES, Kim DW, et al. Empirical treatment of highly suspected nontuberculous mycobacteria infections following aesthetic procedures. Arch Plast Surg 2014;41:759-67.

19. Griffith DE, Aksamit T, Brown-Elliott BA, et al. An official ATS/IDSA statement: diagnosis, treatment, and prevention of nontuberculous mycobacterial diseases. Am J Respir Crit Care Med 2007;175:367-416.

20. Rodriguez JM, Xie YL, Winthrop KL, et al. Mycobacterium chelonae facial infections following injection of dermal filler. Aesthet Surg J 2013; 33:265-9.

21. Chadha R, Grover M, Sharma A, et al. An outbreak of post-surgical wound infections due to Mycobacterium abscessus. Pediatr Surg Int 1998;13:406-10.

22. Nolan CM, Hashisaki PA, Dundas DF. An outbreak of soft-tissue infections due to Mycobacterium fortuitum associated with electromyography. J Infect Dis 1991;163:1150-3.

23. Hall-Stoodley L, Lappin-Scott H. Biofilm formation by the rapidly growing mycobacterial species Mycobacterium fortuitum. FEMS Microbiol Lett 1998;168:77-84. 